\title{
Histeroscopia Cirúrgica com Ressectoscópio para Polipectomia Endometrial: Eficácia e Segurança
}

\author{
Operative Hysteroscopy with Resectoscope for Endometrial Polypectomy: \\ Efficacy and Safety
}

Hélio de Lima Ferreira Fernandes Costa, Arinaldo Vasconcelos de Alencar, Maria do Socorro Alves Carvalho, Silvana Rocha Menelau,

Laura Olinda Bregieiro Fernandes Costa.

\begin{abstract}
RESUM0
Objetivos: avaliar os resultados das primeiras 104 polipectomias histeroscópicas em nossa instituição de ensino.

Métodos: estudo retrospectivo descritivo. Foram revistos os registros das primeiras 136 histeroscopias cirúrgicas realizadas no serviço, sendo 104 polipectomias. Foram avaliadas caracteristicas das pacientes operadas como idade, paridade, fase da vida reprodutiva e sintomatologia; número e tamanho dos pólipos e os resultados em relação às complicações e remissão dos sintomas.

Resultados: as pacientes tinham em média 52,7 anos, sendo três quartos delas multiparas. Encontravam-se na pós-menopausa 60 pacientes (57,7\% do grupo). Cerca de metade das pacientes apresentavam sintomas atribuiveis aos pólipos, sendo o sangramento anormal o mais comum (47,1\%). Em 16,3\% dos casos havia mais de um pólipo, sendo que 84\% deles mediam mais de $1 \mathrm{~cm}$. A única complicação imediata de importância foi uma perfuração uterina, sendo que as complicações tardias foram raras e sem gravidade. O tempo médio de seguimento foi de 9 meses. Em 82\% das pacientes houve remissão das queixas. Apenas 8,2\% das pacientes necessitaram de histerectomia complementar, todas com outras doenças uterinas, como mioma, adenomiose ou hiperplasia endometrial atipica, a qual foi encontrada em apenas 1 paciente. Conclusões: a polipectomia histeroscópica mostrou-se método simples, seguro e eficaz no tratamento dos pólipos endometriais. A seleção das pacientes deve ser rigorosa para evitar procedimentos cirúrgicos complementares.
\end{abstract}

PALAVRAS-CHAVE: Pólipo endometrial. Histeroscopia. Sangramento anormal. Histerectomia.

Introdução

Os pólipos uterinos podem localizar-se na cérvice, na cavidade endometrial ou na transição entre as duas regiões. Com o advento da microhisteroscopia, a freqüência do diagnóstico dos pólipos endometriais aumentou significativamente, assim como o interesse do ginecologista em conhecer melhor essa entidade e suas formas de tratamento. De fato, os pólipos endometriais são

Disciplina de Tocoginecologia da Faculdade de Ciências Médicas da Universidade de Pernambuco. Correspondência:

Hélio de Lima Ferreira Fernandes Costa

Rua Bruno Maia, 217, Apto. 1001 - Graças

52011-110 - Recife - PE encontrados em cerca de um terço das histeroscopias diagnósticas ${ }^{1}$.

Os pólipos endometriais originam-se como uma hiperplasia focal da camada basal do endométrio, dando origem a uma tumoração localizada, recoberta por epitélio. Na constituição dos pólipos encontramos glândulas de aspecto variável, estroma fibrótico e vasos com paredes espessadas. Na dependência do trofismo do componente glandular dos pólipos, são classificados em atróficos, funcionais e hiperplásicos ${ }^{2}$.

Os pólipos acometem as pacientes durante o menacme e na pós-menopausa, sendo raros antes da menarca. Em grande parte são assintomáticos, sendo o sintoma mais freqüente o sangramento, tanto na pré, como na pós-meno- 
pausa $^{3}$. Alguns autores têm comprovado a associação dos pólipos endometriais com infertilidade ${ }^{4,5}$. A associação dos pólipos endometriais com o câncer do endométrio ainda é controversa. Enquanto alguns relataram ocorrência de carcinoma em apenas $0,5 \%$ dos casos $^{6}$, outros observaram, em estudo prospectivo, desenvolvimento de carcinoma em 3,5\% das portadoras de pólipos ${ }^{7}$.

Antes do advento da histeroscopia cirúrgica, os pólipos endometriais eram tratados por curetagem ou histerectomia, a primeira comprovadamente ineficaz ${ }^{8}$ e a segunda, inaceitavelmente agressiva.

Embora aceita, hoje em dia, como o método de eleição para o tratamento dos pólipos endometriais, persistem dúvidas sobre vários aspectos da histeroscopia cirúrgica. Há carência de dados sobres os riscos de complicações, especificamente na polipectomia, em particular, quando realizadas por cirurgiões inexperientes em treinamento. Ademais, alguns autores ${ }^{9,10}$ questionam a eficácia da exérese das lesões intracavitárias (pólipos e miomas) na cura do sangramento uterino anormal, sugerindo que, em muitos casos, a causa do sangramento pode ser outra. O presente estudo visa avaliar a segurança e os resultados da ressectoscopia no tratamento dos pólipos endometriais.

\section{Pacientes e Métodos}

Foi realizado um estudo retrospectivo de caráter descritivo. Foram selecionadas as pacientes submetidas à polipectomia histeroscópica dentre as primeiras 136 histeroscopias cirúrgicas realizadas no Centro Integrado de Saúde Amaury de Medeiros (CISAM) da Maternidade Escola da Faculdade de Ciências Médicas da Universidade de Pernambuco entre janeiro de 1997 e janeiro de 2000.

As pacientes foram encaminhadas para realizar histeroscopia diagnóstica em razão de uma ou mais das seguintes indicações: queixa de sangramento uterino anormal, alterações do eco endometrial à ultra-sonografia, infertilidade ou pólipos endocervicais.

Foram considerados critérios de inclusão: diagnóstico de pólipo endometrial por meio de histeroscopia diagnóstica, ter se submetido à histeroscopia cirúrgica para polipectomia, ter retornado ao serviço pelo menos uma vez após a cirurgia e confirmação do diagnóstico de pólipo endometrial pelo exame histopatológico. Foi considerada como critério de exclusão a impossibilidade de recuperação dos dados do prontuário mé- dico.

Participaram do estudo um total de 104 pacientes, das quais 94 foram submetidas a polipectomias simples e 10 associadas com ablação endometrial. A ablação endometrial era realizada apenas em pacientes no menacme, com prole definida, com mais de 35 anos e com sangramento clinicamente importante.

Não houve qualquer tipo de preparo endometrial prévio. Nenhuma paciente foi operada durante a menstruação. Entretanto, não houve agendamento das cirurgias para alguma época específica do ciclo menstrual.

As cirurgias foram realizadas por dois docentes do serviço ou por residentes de terceiro ano supervisionados por aqueles. Utilizou-se um ressectoscópio de $9 \mathrm{~mm}$ de diâmetro externo (Endoview ${ }^{\mathrm{MR}}$ ) após dilatação do colo com velas de Hegar. A cavidade uterina era distendida com manitol a 3\%, sendo os pólipos removidos por fatiamento eletrocirúrgico com eletrodo do tipo alça. Nos pólipos volumosos a técnica do fatiamento era combinada com a apreensão e tração com pinça de Winter após secção da base dos pólipos com o ressectoscópio. Antibioticoprofilaxia com dose única de $1 \mathrm{~g}$ de cefalotina era empregada rotineiramente.

Revisaram-se os prontuários médicos das pacientes e as fichas de descrição cirúrgica, levantando-se dados a respeito da idade, paridade, fase da vida reprodutiva, sintomatologia, aspecto ecográfico do endométrio, número e tamanho dos pólipos, procedimentos complementares, remissão da sintomatologia e complicações imediatas e tardias.

As informações foram armazenadas e manipuladas no banco de dados Microsoft Access 97 e os resultados expostos sob a forma de tabelas.

A análise estatística consistiu no cálculo de médias, desvios-padrão e percentuais.

O trabalho foi aprovado pelo Comitê de Ética em Pesquisa do CISAM.

\section{Resultados}

A média de idade das pacientes foi de 52,7 ( \pm $11,9)$ anos, sendo a paciente mais jovem de 22 anos e a mais idosa de 82 anos. Encontravam-se na pósmenopausa 60 pacientes $(57,7 \%)$, ao passo que 44 $(42,7 \%)$ estavam no menacme. Três em cada quatro portadoras de pólipo (78 pacientes) já haviam tido 2 ou mais filhos, $14(13,5 \%)$ eram nuliparas e $12(11,5 \%)$ eram primiparas.

Cerca de metade das pacientes $(51,9 \%)$ eram assintomáticas. O sintoma mais freqüente foi o 
sangramento genital, presente em $47 \%$ dos casos, considerando-se suas diversas formas clinicas (Tabela 1). Das 44 pacientes na pré-menopausa, $35(79,5 \%)$ tinham queixa de desvio menstrual para mais, ao passo que das 60 pacientes menopausadas apenas $14(23,3 \%)$ apresentavam sangramento.

Tabela 1 - Distribuição das pacientes submetidas a polipectomia histeroscópica de acordo com a fase da vida reprodutiva.

\begin{tabular}{lcc}
\hline Fase Reprodutiva & $\mathbf{n}$ & $\%$ \\
\hline Menacme & 26 & 25,0 \\
Perimenopausa & 18 & 17,3 \\
Menopausa & 60 & 57,7 \\
Total & 104 & 100 \\
\hline
\end{tabular}

Apenas $38,5 \%$ das pacientes tinham diagnóstico ecográfico prévio de pólipo. Considerandose apenas as 90 pacientes que realizaram ecografia prévia, 94,4\% tinham endométrio anormal ao ultra-som, embora, em $53 \%$ dos casos, a alteração fosse apenas um espessamento inespecífico do eco endometrial (Tabela 2).

Tabela 2 - Distribuição das pacientes submetidas a polipectomia histeroscópica de acordo com a paridade.

\begin{tabular}{lcc}
\hline Paridade & $\mathbf{n}$ & $\%$ \\
\hline 0 & 14 & 13,5 \\
1 & 12 & 11,5 \\
$\geq 2$ & 78 & 75,0 \\
Total & 104 & 100 \\
\hline
\end{tabular}

Em 80 pacientes $(76,9 \%)$ havia um único pólipo, em 10 delas $(9,6 \%)$ havia 2 pólipos e em 7 $(6,7 \%)$ coexistiam 3 ou mais pólipos. Dos 84 pólipos mensurados pela histeroscopia, $79,8 \%$ eram maiores que $1 \mathrm{~cm}$. Mediam mais de $3 \mathrm{~cm}$ no seu maior diâmetro $11(10,6 \%)$ dos pólipos.

Como complicações imediatas anotamos apenas um caso de perfuração uterina sem lesões de vísceras adjacentes. Em um tempo médio de seguimento de 9 meses foram verificadas 4 complicações tardias de pequena gravidade. Duas pacientes que foram submetidas a ablação endometrial concomitante desenvolveram dismenorréia, sendo que em uma delas foi realizada a histerectomia, revelando a presença de adenomiose. Uma paciente desenvolveu endometrite e outra apresentou sinéquias uterinas em histeroscopia de controle. Em todas as pacientes a exérese do pólipo foi realizada de maneira completa, com exceção daquela na qual houve a perfuração uterina.

Das 49 pacientes com queixa inicial de sangramento, 81,6\% tiveram resolução de seu sintoma após a polipectomia. Em apenas 4 pacientes o sangramento persistiu e motivou a realização de histerectomia, sendo que, em todos os casos, foram encontradas outras afecções uterinas que justificavam o sangramento, conforme revela a Tabela 3. Nos exames histopatológicos dos pólipos, identificou-se um caso de hiperplasia endometrial atípica não suspeitada previamente.

Tabela 3 - Distribuição das pacientes submetidas a polipectomia histeroscópica de acordo com a sintomatologia.

\begin{tabular}{lrr}
\hline Sintomas & $\mathbf{n}$ & $\mathbf{\%}$ \\
\hline Hipermenorréia/ragia & 33 & 31,7 \\
Metrorragia & 16 & 15,4 \\
Dismenorréia & 4 & 3,8 \\
Infertilidade & 2 & 1,9 \\
Assintomática & 54 & 51,9 \\
\hline
\end{tabular}

Base: 104 pacientes.

Obs: Algumas pacientes tinham mais de 1 sintoma

\section{Discussão}

A maioria das pacientes eram menopausadas, sendo a média de idade de 52 anos. A média de idade das pacientes deste estudo é um pouco mais alta do que a relatada em casuísticas mais antigas. De fato, Peterson e Novak ${ }^{11}$ encontraram o pico de incidência dos pólipos entre os 40 e 49 anos. Esse dados reportam-se à época em que não se dispunha da ultra-sonografia transvaginal e em que o diagnóstico dos pólipos quase sempre só era firmado em material de curetagem ou histerectomia realizadas por sangramento uterino anormal. Assim, grande parte dos pólipos assintomáticos de mulheres menopausadas provavelmente não eram descobertos. O achado de $75 \%$ das portadoras de pólipo terem tido 2 ou mais gestações é condizente com outros autores, que reafirmam a maior freqüência dos pólipos entre multíparas ${ }^{12}$.

A maioria dos pólipos em nossa casuística era assintomática. $\mathrm{O}$ sintoma predominante foi o sangramento genital, presente em $47 \%$ dos casos. Predominou entre as nossas pacientes o sangramento do tipo hipermenorréia ou hipermenorragia, ao contrário do que relatam outros autores ${ }^{13}$ que afirmam ser a metrorragia ou 
os spottings pré e pós-menstruais a modalidade mais freqüente de sangramento. Os vasos que irrigam os pólipos, embora espessos, são axiais e portanto protegidos de eventuais traumas que provoquem metrorragia. $\mathrm{O}$ aumento da superficie endometrial sangrante é uma explicação plausível para a hipermenorréia e menorragia, ao passo que a necrose das porções distais dos pólipos poderia justificar os episódios de metrorragia. A proporção de pólipos assintomáticos está em relação direta com o status menopausal. Nossos dados indicam que durante o menacme a probabilidade de os pólipos endometriais serem sintomáticos é cerca de 3 vezes maior que após a menopausa. Achados de Reslová et al. ${ }^{14}$ em 1999 reforçam nossa observação.

Dentre as pacientes com ecografia prévia, cerca de $95 \%$ apresentavam anormalidade endometrial identificável. Entretanto, em mais de metade dos casos o achado ecográfico era inespecífico, no sentido de revelar apenas espessamento endometrial. Soares et al. ${ }^{15}$ relatam uma sensibilidade de $75 \%$ no diagnóstico de lesões polipóides à ecografia transvaginal. Stadtmauer e Grunfeld ${ }^{16}$ observam que a identificação dos pólipos endometriais à ecografia é melhor se o exame é realizado na primeira fase do ciclo menstrual, tendo em vista que os pólipos como estruturas predominantemente hiperecogênicas se destacariam facilmente do endométrio proliferativo hipoecóico. Nesse sentido é fundamental observar a fase do ciclo em que se programam as ecografias transvaginais, visto que a "janela" que a fase proliferativa propicia para o exame é curta, devido ao sangramento menstrual dos primeiros dias dessa fase. Durante a menopausa, o endométrio além de fino, é costumeiramente hiperecogênico, o que dificultaria a identificação dos pólipos.

A polipectomia histeroscópica revelou-se um método seguro nessa casuística. A única complicação transoperatória observada foi um caso de perfuração uterina durante a dilatação cervical com velas de Hegar em paciente menopausada e colo semi-estenosado. O procedimento foi imediatamente interrompido, não havendo lesão de visceras adjacentes. A paciente foi submetida a histerectomia total abdominal no mesmo ato, sem complicações.

O intravasamento do fluido distensor levando à síndrome da hipervolemia/intoxicação hídrica tem sido apontado como o maior risco isolado das histeroscopias cirúrgicas para remoção de lesões intracavitárias ${ }^{10}$. Não registramos nenhum caso em nossa série de polipectomias. Sem dúvida a polipectomia oferece menores riscos que a miomectomia, que em geral é procedimento mais demorado. Acreditamos que outro fator importan- te para a não-ocorrência de hipervolemia ou intoxicação hídrica decorrentes do intravasamento tenha sido a utilização de manitol como meio distensor, dada sua ação diurética quando entra na corrente circulatória.

O surgimento ou agravamento de dismenorréia foi a principal complicação tardia, ocorrendo em duas pacientes, ambas com ablação endometrial concomitante. Esse tipo de complicação da ablação endometrial pode, em alguns casos, estar associada ao desenvolvimento de uma endometrite granulomatosa ${ }^{17}$. Entretanto, na maioria das vezes, tem como substrato anatômico a adenomiose ${ }^{18}$, conforme encontrado na paciente da nossa série submetida à histerectomia. No momento, ainda é controverso se a ablação endometrial predispõe ao surgimento de adenomiose ou se esses casos representam a evolução natural de adenomiose previamente instalada. Mencaglia e Tantini ${ }^{19}$ em 1996 descreveram um caso de adenomiose de rápido desenvolvimento após miomectomia histeroscópica. Hallez ${ }^{20}$ sugere que as sinéquias que podem surgir após a ablação endometrial induzam o aparecimento da adenomiose. É possivel que o trauma miometrial decorrente da histeroscopia cirúrgica possa de fato predispor à adenomiose. Uma de nossas pacientes desenvolveu endometrite clínica leve, resolvida com antibioticoterapia, e outra apresentou uma sinéquia uterina em histeroscopia de controle, sem repercussão clínica.

A resolução do sangramento anormal após um tempo médio de seguimento de 9 meses foi observada em $81,6 \%$ das pacientes. O pequeno número de ablações endometriais realizadas e o fato de terem sido empreendidas preferencialmente nas pacientes com sangramento mais importante não permitem conclusões a respeito de ganho de resolutividade associado à sua realização. Loffer ${ }^{10}$, entretanto, afirma que a taxa de controle do sangramento é significativamente aumentada quando se realiza ablação endometrial concomitante.

A comparação dos nossos resultados com os da literatura tem algumas limitações. De início, o curto tempo de seguimento não permite conclusões definitivas. Ademais, a maioria dos trabalhos da literatura relatam os resultados de exérese de lesões intracavitárias, englobando indistintamente os miomas e os pólipos. Derman et al. ${ }^{21}$, em seguimento de mais de 8 anos, encontrou uma taxa de persistência ou recorrência do sangramento de $24,5 \%$, com necessidade de nova cirurgia em $15,9 \%$ das pacientes. Loffer ${ }^{22}$, por sua vez, conseguiu normalizar o sangramento excessivo em 12 de 95 pacientes submetidas a polipectomia ou miomectomia histeroscópica por essa queixa. 
Em nossa casuística, das 9 pacientes que persistiram com sangramento, apenas 4 necessitaram de histerectomia, sendo que todas elas tinham outro achado uterino que justificava o sangramento e que não era passivel de resolução pela via histeroscópica. As demais 5 pacientes obtiveram uma melhora apenas parcial do sangramento, porém não necessitaram de tratamento complementar. As afecções encontradas nas peças das histerectomias das nossas pacientes provavelmente já existiam no momento da polipectomia. Seus diagnósticos não foram possíveis no pré-operatório (adenomiose e hiperplasia atípica) ou não foram valorizados como causa do sangramento (mioma).

A histeroscopia cirúrgica, portanto, mostrouse um método seguro e eficaz no tratamento dos pólipos endometriais.

\section{SUMMARY}

Purpose: to evaluate the results of the first 104 hysteroscopic polypectomies in a teaching hospital.

Methods: a retrospective descriptive study was designed. Medical records of the first 136 operative hysteroscopies 104 of which polypectomies - were reviewed. Patient characteristics such as age, parity, period of reproductive function and symptoms; number and size of polyps and results concerning complications and symptom relief were evaluated.

Results: the average age of patients was 52.7 years. Three quarters of them were multiparous. Fifty-seven percent of the patients were menopaused. About half of the patients had symptoms related to polyps. Abnormal bleeding was the most frequent symptom (47.1\%). In 16.3\% of the patients more than 1 polyp were detected and $84 \%$ of the polyps were larger than $1 \mathrm{~cm}$. The only immediate complication was a uterine perforation. Late complications were rare and mild. The follow-up period was 9 months on average. In $82 \%$ of teh patients the symptoms were controlled. Hysterectomy was necessary in $8.2 \%$ of the patients, all of them with other uterine diseases such as leiomyomas, adenomyosis and atypical endometrial hyperplasia in one patient).

Conclusion: hysteroscopic polypectomy is a simple, safe and effective method for the treatment of endometrial polyps. Selection of patients must be rigorous to avoid further operative procedures.

KEY WORDS: Endometrial polyp. Hysteroscopy. Abnormal uterine bleeding. Hysterectomy.

\section{Referências}

1. Fabres C, Alam V, Balmaceda J, Zegers-Hochschild F, Mackenna A, Fernandez E. Comparison of ultrasonography and hysteroscopy in the diagnosis of intrauterine lesions in infertile women. J Am Assoc Gynecol Laparosc 1998; $5: 375-8$.

2. Pessini SA. Lesões precursoras do câncer de endométrio. In: Oliveira HC, Lemgruber I, Costa OT, editores. Tratado de Ginecologia Febrasgo. $1^{\text {a }}$ ed. Rio de Janeiro: Revinter; 2000. p.1301-6.

3. Nagele F, O'Connor H, Baskett TT, Davies A, Mohammed H, Magos AL. Hysteroscopy in woman with abnormal uterine bleeding on hormone replacement therapy: a comparison with postmenopausal bleeding. Fertil Steril 1996; 65:1145-50.

4. Varasteh NN, Neuwirth RS, Levin B, Keltz MD. Pregnancy rates after hysteroscopic polypectomy and myomectomy in infertile women. Obstet Gynecol 1999; 94:168-71.

5. Lass A, Williams G, Abusheikha N, Brinsden P. The effect of endometrial polyps on outcomes of in vitro fertilization (IVF) cycles. J Assist Reprod Genet 1999; 16:410-5.

6. Kurman RJ, Mazur MT. Benign disease of the endometrium. In: Kurman RJ, editor. Pathology of the Female Genital Tract. $4^{\text {th }}$ ed. New York: Springer-Verlag; 1994. p.367-409.

7. Armenia CS. Sequential relationship between endometrial polyps and carcinoma of the endometrium. Obstet Gynecol 1967; 30:524-9.

8. Bonavolontà G, Rossetti A, Cannella PL, Campo S, Garcea N. Curettage vs. hysteroscopic resection. Minerva Ginecol 1994; 46:1-3.

9. Indman PD. Hysteroscopic treatment of menorrhagia associated with uterine leiomyomas. Obstet Gynecol 1993; 81:716-20.

10.Loffer FD. Removing intrauterine lesions: myomectomy and polypectomy. In: Bieber EJ, Loffer FD, editors. Gynecologic Resectoscopy. $1^{\text {st }}$ ed. Cambridge: Blackwell Science; 1995. p.16894.

11.Peterson WF, Novak ER. Endometrial polyps. Obstet Gynecol 1956; 8:40-9.

12.Dias R, Pacheco JF, Pontes A, Leite NJ, Fontana AL. Pólipos endometriais: uma revisão. Femina 1998; 26:579-81.

13. Marcondes DAP, Fonseca NM. Pólipo do útero II. Anatomia patológica, diagnóstico e tratamento. Femina 1993; 21:689-98.

14. Reslová T, Tosner J, Resl M, Kugler R, Vávrová I. Endometrial polyps. A clinical study of 245 cases. Arch Gynecol Obstet 1999; 262:133-9. 
15. Soares SR, Barbosa dos Reis MM, Camargos AF. Diagnostic accuracy of sonohysterography, transvaginal sonography, and hysterosalpingography in patients with uterine cavity diseases. Fertil Steril 2000; 73:406-11.

16. Stadtmauer L, Grunfeld L. The significance of endometrial filling defects detected on routine transvaginal sonography. J Ultrasound Med $1995 ; 14: 169-72$.

17.Ferryman SR, Stephens J, Gough D. Necrotising granulomatous endometritis following endometrial ablation therapy. $\mathrm{Br} \mathrm{J}$ Obstet Gynaecol 1992; 99:928-30.

18. Magos A. Control of menorrhagia by endometrial resection. In: Bieber EJ, Loffer FD, editors.
Gynecologic Resectoscopy. $1^{\text {st }}$ ed. Cambridge: Blackwell Science; 1995. p.168-94.

19.Mencaglia L, Tantini C. GnRH agonist analogues and hysteroscopic resection of myomas. Int $\mathrm{J}$ Gynecol Obstet 1996; 43:285-8.

20.Hallez JP. Myomectomies by endo-uterine resection. Curr Opin Obstet Gynecol 1996; 8:250-6.

21.Derman SG, Rehnstrom J, Neuwirth RS. The longterm effectiveness of hysteroscopic treatment of menorrhagia and leiomyomas. Obstet Gynecol 1991; 77:591-4.

22.Loffer FD. Removal of large symptomatic intrauterine growths by the hysteroscopic resectoscope. Obstet Gynecol 1990; 76: 836-40.

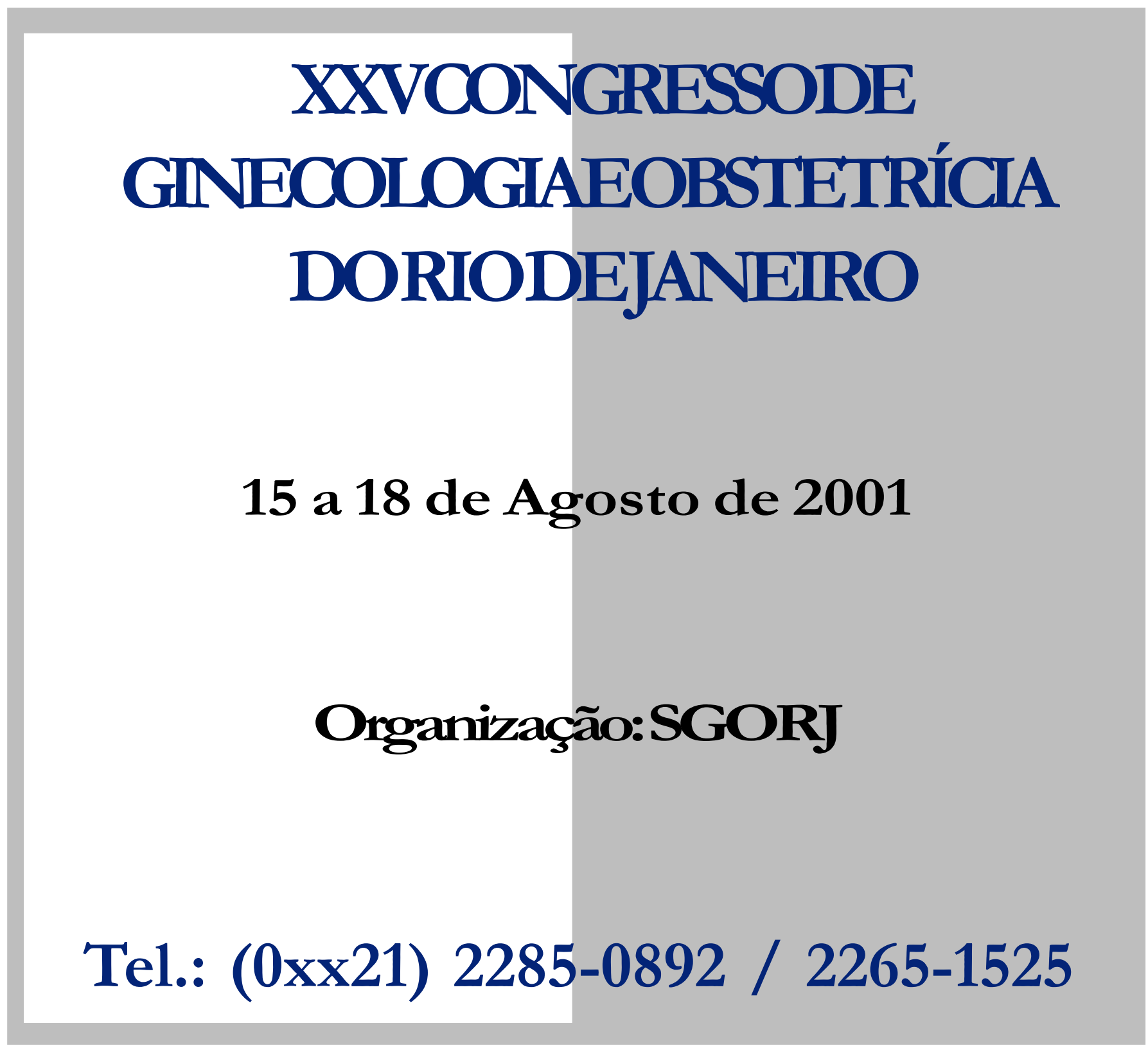

